Proceedings of the 42th "Jaszowiec" International School and Conference on the Physics of Semiconductors, Wisła 2013

\title{
Analysis of Gain-Switching in Two-Section Tapered Lasers
}

\author{
P. AdAmieC*, J.M.G. TiJero And I. Esquivias
}

Universidad Politécnica de Madrid, E.T.S.I. Telecomunicación-CEMDATIC, 28040 Madrid, Spain

\begin{abstract}
We analyze the gain-switching dynamics of two-section tapered lasers by means of a simplified three-rate-equation model. The goal is to improve the understanding of the underlying physics and to optimize the device geometry to achieve high power short duration optical pulses.
\end{abstract}

DOI: $10.12693 /$ APhysPolA.124.888

PACS: 42.55.Px, 42.55.Ah, 07.05.Tp

\section{Introduction}

High power short optical pulses directly generated by semiconductor lasers are of high interest for different applications. The pulses are usually generated by gain switching, mode-locking, or $Q$-switching. Tapered lasers with separate injection of the ridge waveguide (RW) and tapered sections have demonstrated very high $\mathrm{cw}$ power levels [1] and are promising devices for short pulse generation because of the possibility of driving their dynamics by a low current injection in the RW section. In particular, the generation of high power short optical pulses at $1060 \mathrm{~nm}$ by $Q$ - and gain-switching has been demonstrated in these devices [2,3]. $13 \mathrm{~W}$ peak power and 100 ps duration pulses have been reported in master-oscillator power-amplifiers being excited as if they were distributed Bragg reflector (DBR) tapered lasers [4].

We have proposed recently a simple rate equation (RE) model to study the dynamics of two-section tapered lasers [3]. In spite of the simplicity of the model, we have found that it provides good qualitative agreement with the experiments and that it enlightens the complex dynamic interaction between carriers and photons in such devices. In this work we apply the model to the analysis of gain-switching in two-section tapered lasers with different geometries.

\section{Model}

The main idea of this model is to substitute the tapered section by a rectangular section with the same area and length (see the inset in Fig. 1a), assuming carrier and photon uniformity within each section along the lateral and longitudinal directions. The RE can then be expressed as [3]:

$$
\begin{aligned}
\frac{\mathrm{d} n_{i}}{\mathrm{~d} t} & =\frac{\eta_{\mathrm{int}} I_{i}}{q V_{i}}-R\left(n_{i}\right)-\nu_{\mathrm{g}} g\left(n_{i}\right) a_{i} S_{0}, \quad i=1,2, \\
\frac{\mathrm{d} S_{0}}{\mathrm{~d} t} & =\frac{\Gamma \nu_{\mathrm{g}}}{L_{\mathrm{tot}}}\left[L_{1} g\left(n_{1}\right)+L_{2} g\left(n_{2}\right)\right] S_{0} \\
& -\frac{S_{0}}{\tau_{p}}+\beta R_{s p}\left(n_{1}, n_{2}\right),
\end{aligned}
$$

*corresponding author; e-mail: pawel.adamiec@tfo.upm.es where the subscript $i$ refers to the RW section $(i=1)$ and tapered section $(i=2)$, respectively, $R(n)$ is the recombination rate, which takes into account non-radiative, bimolecular, and Auger recombination, $S_{0}$ is the average photon density along the complete device and other symbols have their usual meanings (see Ref. [3]). The material gain $g(n)$ is modeled using a modified logarithmic function [5] and a standard gain compression term and reads

$$
\begin{aligned}
& g\left(n_{i}\right)=\frac{g_{0}}{1+\varepsilon a_{i} S_{0}}[\arctan u \\
& \left.-2 \arctan \left(u-\frac{n_{i}}{n_{\mathrm{tr}}}\right)-\frac{\pi}{2}\right],
\end{aligned}
$$

where $\varepsilon$ is the non-linear gain coefficient, $u$ is the frequency shift, and $n_{\mathrm{tr}}$ is the transparency density. The coefficients $a_{i}$ account for the difference in the photon densities of the two sections. Assuming continuity of the photon number at the interface between the two sections, they are given by $a_{i}=A_{\mathrm{tot}} w_{i}^{-1} L_{\mathrm{tot}}^{-1}$, where $A_{\mathrm{tot}}$ is the overall area of the tapered laser, so the ratio between the photon densities in the RW and tapered sections is then $a_{1} / a_{2}=w_{2} / w_{1}$.

TABLE I

Simulation parameters.

\begin{tabular}{l|c}
\hline \hline \multicolumn{1}{c}{ Parameter } & Value \\
\hline optical confinement factor $\Gamma$ & $7.8 \times 10^{-3}$ \\
spontaneous emission factor $\beta$ & $1 \times 10^{-6}$ \\
non-linear gain coefficient $\varepsilon\left[\mathrm{m}^{3}\right]$ & $1.32 \times 10^{-24}$ \\
differential gain $g_{0}\left[\mathrm{~m}^{-1}\right]$ & $2.45 \times 10^{5}$ \\
transparency density $n_{\mathrm{tr}}\left[\mathrm{m}^{-3}\right]$ & $2.6 \times 10^{24}$ \\
frequency detuning $u$ & $1 \times 10^{-3}$ \\
non-radiative coefficient $A\left[\mathrm{~s}^{-1}\right]$ & $7.2 \times 10^{8}$ \\
bimolecular coefficient $B\left[\mathrm{~m}^{3} \mathrm{~s}^{-1}\right]$ & $0.36 \times 10^{-16}$ \\
Auger coefficient $C\left[\mathrm{~m}^{6} \mathrm{~s}^{-1}\right]$ & $1.58 \times 10^{-42}$
\end{tabular}

The model has been applied to $980 \mathrm{~nm}$ two-section tapered lasers with geometry and material parameters similar to those reported in Ref. [6]. In order to understand qualitatively the influence of the geometry in the gain-switching process, we will compare in the following the results for fixed values of $w_{1}=3 \mu \mathrm{m}$ and $L_{1}=1.35 \mathrm{~mm}$, and different values of $w_{2}(3 \mu \mathrm{m}, 30 \mu \mathrm{m}$ and $150 \mu \mathrm{m})$, which is equivalent to modify the taper an- 


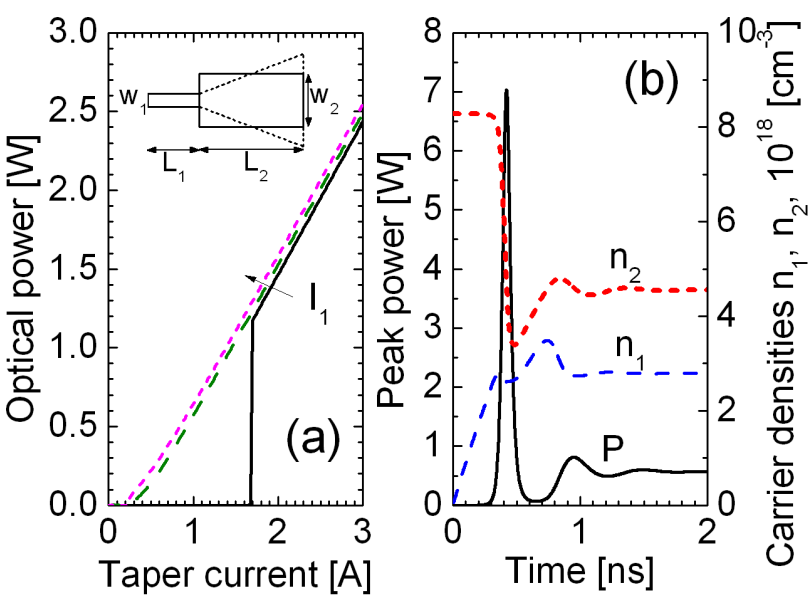

Fig. 1. (a) Optical power vs. tapered section current for RW currents $I_{1}=0 \mathrm{~mA}, 50$ and $100 \mathrm{~mA}$. The inset illustrates the geometrical parameters and the simplification used in the model. (b) Dynamics of optical power and carrier densities when switching on the RW current. See text for details. The geometry is $L_{2} / L_{1}=2$, $w_{2}=30 \mu \mathrm{m}$.

gle, and $L_{2}\left(L_{2} / L_{1}\right.$ equal to 1,2 , and 3$)$. The parameters used in simulation can be found in [6] and Table I.

\section{Results}

The power-tapered section current $\left(P-I_{2}\right)$ characteristics at constant RW current $\left(I_{1}\right)$ were obtained by solving the rate equations for a time window long enough to achieve steady state. As an example, they are shown in Fig. 1a for $w_{2}=30 \mu \mathrm{m}$ and $L_{2} / L_{1}=2$. The tapered section current required to achieve threshold when $I_{1}=0$, defined as $I_{2 \operatorname{th}}(0)$, is around $1.7 \mathrm{~A}$, much higher than the value required when the RW is injected, $I_{2 \text { th }} \approx 0.3 \mathrm{~A}$. This is a consequence of the high losses of the non-injected RW section. At threshold, the optical power jumps abruptly to a value similar to that of the cases when $I_{1} \neq 0$, due to the bleaching of the RW section. This behavior is typical of two-section lasers and produces a bistable $P-I_{2}$ characteristic [7]. The values of $I_{2 \text { th }}(0)$ for other geometries are shown in Table II. As expected, $I_{2 \text { th }}(0)$ increases with $w_{2}$, due to the larger area, and decreases when $L_{2} / L_{1}$ increases due to the reduction of the relative contribution of the RW section to the gain.

\section{TABLE II}

Threshold currents $I_{2 \operatorname{th}}(0)$ for different geometries (in A).

\begin{tabular}{l|c|c|c}
\hline \hline & $L_{2} / L_{1}=1$ & $L_{2} / L_{1}=2$ & $L_{2} / L_{1}=3$ \\
\hline$w_{2}=3 \mu \mathrm{m}$ & 13.94 & 0.32 & 0.12 \\
$w_{2}=30 \mu \mathrm{m}$ & 16.41 & 1.70 & 1.00 \\
$w_{2}=150 \mu \mathrm{m}$ & 20.85 & 5.26 & 4.30
\end{tabular}

An example of the gain-switching dynamics is shown in Fig. 1b. The tapered section is driven in $\mathrm{cw}$ at a current lower than $I_{2 \operatorname{th}}(0)$ and at $t=0, I_{1}$ is switched on from 0 to $50 \mathrm{~mA}$. As a result of it, the carrier density in the RW section $n_{1}$ builds up until the threshold condition is reached. Then a high power short pulse $(\approx 7 \mathrm{~W}$ and $\approx 70 \mathrm{ps}$ ) is emitted, the carrier density in the tapered section $n_{2}$ decreases, and after some relaxation oscillations the steady-state is reached again.

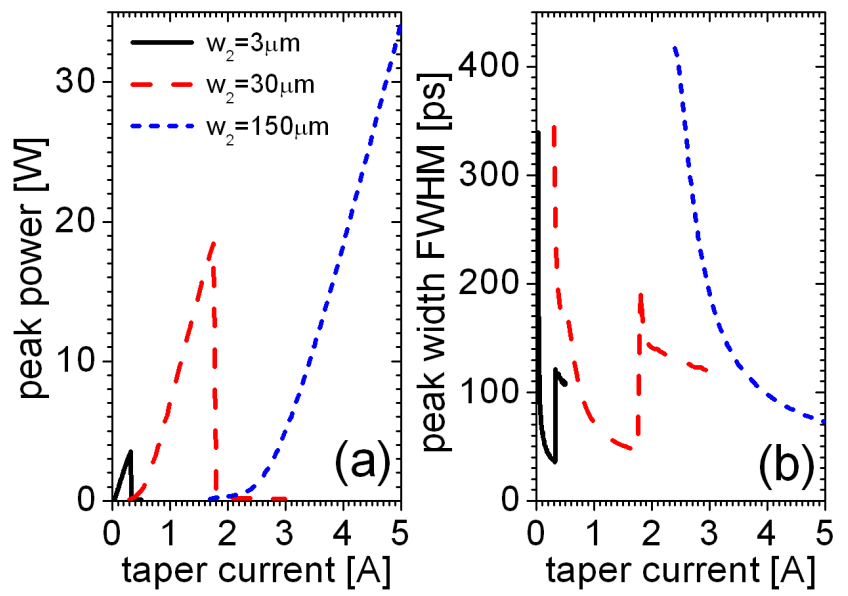

Fig. 2. Peak power (a) and pulse FWHM (b) vs. tapered section current for devices with constant $L_{2} / L_{1}=2$, and different $w_{2}$. $\quad I_{1}$ switches from 0 to $50 \mathrm{~mA}$.

We analyze now the dependence of the peak power $P_{\text {peak }}$ and pulse FWHM $\Delta \tau$ on the driving conditions and device geometry. Figure 2 shows $P_{\text {peak }}$ and $\Delta \tau$ as a function of $I_{2}$ for devices with $L_{2} / L_{1}=2$ and different values of $w_{2}$. For $I_{2}<I_{2 \operatorname{th}}(0)$, the laser is initially in the off state, and $P_{\text {peak }}$ increases and $\Delta \tau$ decreases when $I_{2}$ increases. For $I_{2}>I_{2 \text { th }}(0)$, the laser is initially on and there is no gain-switching but a simple direct modulation. A similar behavior was experimentally observed in [3]. The simulations predict pulses as short as $\approx 50 \mathrm{ps}$ when driving the tapered section at a current close but lower than $I_{2 \text { th }}(0)$ in the narrowest laser, and slightly longer pulses for broader lasers.

The pulse parameters obtained in devices with different $L_{2} / L_{1}$ ratio are shown in Fig. 3 . The same trends of $P_{\text {peak }}$ and $\Delta \tau$ as a function of $I_{2}$ can be observed. The maximum power and minimum pulse width were obtained for $L_{2} / L_{1}=2$ within a reasonable current range, as for $L_{2} / L_{1}=1, I_{2 \mathrm{th}}(0)$ is extremely high for practical applications (see Table II). It is important to remark that by optimizing the driving conditions and geometry, for instance in point $M$ in Fig. 3, it is possible to obtain peak power 20 times higher than the corresponding steady state output power, with pulse duration in the tens of ps range, and that this can be achieved by switching on a relatively small current. It is worth to mention that in similar devices the experimentally obtained peak power was 8-12 times higher than the corresponding steady state values [3]. 

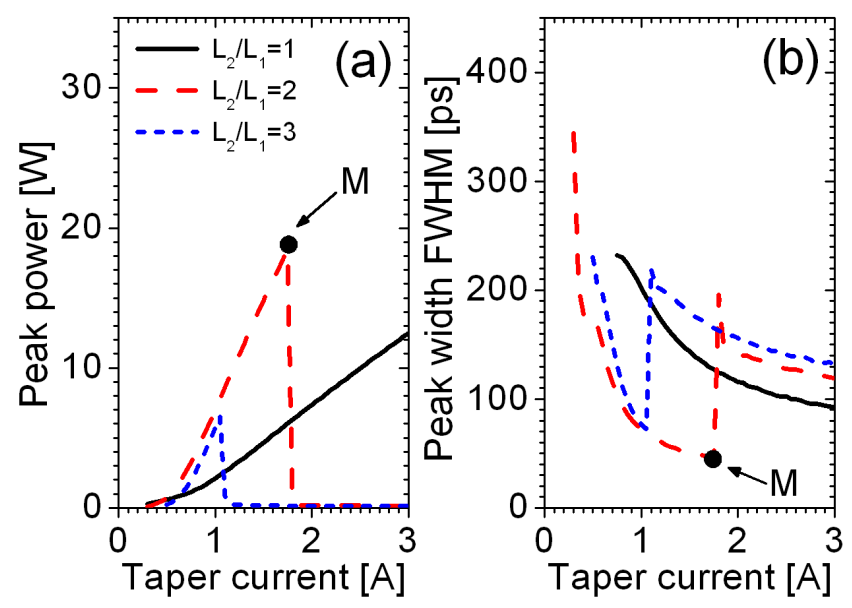

Fig. 3. Peak power (a) and pulse FWHM (b) vs. tapered section current for devices with constant $w_{2}=$ $30 \mu \mathrm{m}$ and different $L_{2} / L_{1}$, switching as in Fig. 2.

\section{Summary}

The results of the rate equation model provide a qualitative understanding of the gain-switching dynamics of two-section tapered lasers and indicate that the peak power and pulse width can be optimized by adjusting the driving conditions and geometrical design. Although the simplicity of the model does not allow quantitative predictions, it is clear that these devices are a promising approach to produce high peak power short optical pulses.

\section{Acknowledgments}

This work has been founded by Ministerio de Ciencia e Innovacion de España within the project TEC2012-38864, and European program PEOPLE - Marie Curie IEF, project No. E100945552.

\section{References}

[1] B. Sumpf, K.-H. Hasler, P. Adamiec, F. Bugge, F. Dittmar, J. Fricke, H. Wenzel, M. Zorn, G. Erbert, G. Trankle, IEEE J. Sel. Top. Quantum Electron. 15, 1009 (2009).

[2] A. Klehr, B. Sumpf, K.-H. Hasler, J. Fricke, A. Liero, T. Hoffmann, G. Erbert, G. Trankle, Proc. SPIE 7616, 76161J (2010).

[3] P. Adamiec, A. Consoli, J.M.G. Tijero, I. Esquivias, S. Schwertfeger, A. Klehr, H. Wenzel, G. Erbert, Proc. SPIE 8277, 82771N (2012).

[4] H. Wenzel, S. Schwertfeger, A. Klehr, D. Jedrzejczak, T. Hoffmann, G. Erbert, Opt. Lett. 37, 1826 (2012).

[5] S. Balle, Phys. Rev. A 57, 1304 (1998).

[6] H. Odriozola, J.M.G. Tijero, L. Borruel, I. Esquivias, H. Wenzel, F. Dittmar, K. Paschke, B. Sumpf, G. Erbert, IEEE J. Quantum Electron. 45, 42 (2009).

[7] G.H. Duan, P. Landais, J. Jacquet, IEEE J. Quantum Electron. 30, 2507 (1994). 